\title{
Statistical Ensembles with Volume Fluctuations
}

\author{
Mark I. Gorenstein ${ }^{1}$ \\ ${ }^{1}$ Bogolyubov Institute for Theoretical Physics, Kiev, Ukraine
}

\begin{abstract}
The volume fluctuations in statistical mechanics are discussed. First, the volume fluctuations in ensembles with a fixed external pressure, the so called pressure ensembles, are considered. Second, a generalization of the pressure ensembles is suggested. Namely, the statistical ensembles with the volume fluctuating according to externally given distributions are considered. Several examples and possible applications in statistical models of hadron production are discussed.
\end{abstract}

PACS numbers: 24.10.Lx, 24.60.Ky, 25.75.-q

Keywords: statistical ensembles, pressure ensembles, volume fluctuations, particle number fluctuations 


\section{INTRODUCTION}

Successful application of the statistical model to description of mean hadron multiplicities in high energy collisions (see, e.g., recent papers [1] and references therein) has stimulated investigations of properties of statistical ensembles. Whenever possible, one prefers to use the grand canonical ensemble (GCE) due to its mathematical convenience. The canonical ensemble (CE) [2] should be applied when the number of carriers of a conserved charges is small (of the order of 1), such as strange hadrons [3], anti-baryons [4], or charmed hadrons [5]. The micro-canonical ensemble (MCE) [6] has been used to describe small systems with fixed energy, e.g. mean hadron multiplicities in proton-antiproton annihilation at rest. In all these cases, calculations performed in different statistical ensembles yield different results. This happens because the systems are 'small' and they are 'far away' from the thermodynamic limit (TL). The mean multiplicity of hadrons in relativistic heavy ion collisions ranges from $10^{2}$ to $10^{4}$, and mean multiplicities (of light hadrons) obtained within GCE, CE, and MCE approach each other. One refers here to the thermodynamical equivalence of statistical ensembles and uses the GCE for calculating the hadron yields.

Measurements of a hadron multiplicity distribution $P(N)$ in interactions, including nucleusnucleus collisions, open a new field of applications of the statistical models. The particle multiplicity fluctuations are usually quantified by the ratio of variance to mean value of a multiplicity distribution $P(N)$, the scaled variance, and are a subject of current experimental activities. In statistical models there is a qualitative difference in the properties of mean multiplicity and scaled variance of multiplicity distributions. It was recently found [7, $\underline{8}, \underline{9}, \underline{10}$, 11, 12, 13] that even in the TL corresponding results for the scaled variance are different in different ensembles. Hence the equivalence of ensembles holds for mean values in the TL, but does not extend to fluctuations.

A statistical system is characterized by the extensive quantities: volume $V$, energy $E$, and conserved charge $(\mathrm{s})^{1} Q$. The MCE is defined by the postulate that all micro-states with given $V, E$, and $Q$ have equal probabilities of being realized. This is the basic postulate of the statistical mechanics. The MCE partition function just calculates the number of microscopic

\footnotetext{
${ }^{1}$ In statistical description of the hadron or quark-gluon systems, these conserved charges are usually the net baryon number, strangeness, and electric charge. In non-relativistic statistical mechanics, the number of particles plays the role of a conserved 'charge'.
} 
states with given fixed $(V, E, Q)$ values. In the CE the energy exchange between the considered system and 'infinite thermal bath' is assumed. Consequently, a new parameter, temperature $T$ is introduced. To define the GCE, one makes a similar construction for conserved charge $Q$. An 'infinite chemical bath' and the chemical potential $\mu$ are introduced. The CE introduces the energy fluctuations. In the GCE, there are additionally the charge fluctuations. The $\mathrm{MCE}, \mathrm{CE}$, and GCE are the most familiar statistical ensembles. In several textbooks (see, e.g., Ref. [14, 15]), the pressure (or isobaric) canonical ensemble has been also discussed. The 'infinite bath of the fixed external pressure' $p_{0}$ is then introduced. This leads to the volume fluctuations around the average value.

A more general concept of the statistical ensembles was suggested in Ref. [16]. The statistical ensemble is defined by an externally given distribution of extensive quantities, $P_{\alpha}(\vec{A})$. The construction of distribution of any property $O$ in such an ensemble proceeds in two steps. Firstly, the MCE $O$-distribution, $P_{m c e}(O ; \vec{A})$, is calculated at fixed values of the extensive quantities $\vec{A}=(V, E, Q)$. Secondly, this result is averaged over the external distribution $P_{\alpha}(\vec{A})$ [16],

$$
P_{\alpha}(O)=\int d \vec{A} P_{\alpha}(\vec{A}) P_{m c e}(O ; \vec{A})
$$

The ensemble defined by Eq. (1), the $\alpha$-ensemble, includes the standard statistical ensembles as particular cases.

Recently, the micro-canonical ensemble with the volume fluctuations was introduced [17] for modelling the hadron production in high energy interactions. An introduction of the volume fluctuations was necessary in order to reproduce the KNO-scaling [18] of the hadron multiplicity distribution observed in proton-proton and proton-antiproton collisions at high energies. The volume fluctuations lead also to the power law shape of the single particle spectra at high transverse momenta.

In the present paper the statistical ensembles with volume fluctuations are studied. First, the pressure ensembles are considered. In general, there are 3 pairs $^{2}$ of variables -

2 In the present study we do not discuss the role of the total 3-momentum. As shown in Ref. [13] the total momentum conservation is not important in the TL for thermodynamical functions and fluctuations in the full phase space. It may however influence the particle number fluctuations in the limited segments of the phase space. 
$\left(V, p_{0}\right),(E, T),(Q, \mu)$ - and, thus, the 8 statistical ensembles ${ }^{3}$ can be constructed. Among these 8 ensembles there are 4 pressure ensembles: $\left(p_{0}, E, Q\right), \quad\left(p_{0}, T, Q\right),\left(p_{0}, E, \mu\right)$, and $\left(p_{0}, T, \mu\right)$. In addition to the pressure canonical ensemble known from the literature, three other possibilities - pressure micro-canonical, pressure grand micro-canonical, and pressure grand canonical ensembles - are constructed and studied. In Section II, non-relativistic statistical systems are discussed, whereas Section III presents the results for an ultra-relativistic ideal gas.

In Section IV the concept of pressure ensembles is extended to the case of more general volume fluctuations. Namely, the statistical ensembles with volume fluctuating according to externally given distributions are introduced. The Summary presented in Section V closes the paper.

\section{NON-RELATIVISTIC BOLTZMANN GAS}

\section{A. Canonical and Micro-Canonical Ensembles}

In this Section the system of non-relativistic Boltzmann particles is discussed. The $(V, T, N)$ Canonical Ensemble (CE) partition function of $N$ particles reads [19]:

$$
Z_{c e}(V, T, N)=\frac{1}{N !} \int \frac{d \mathbf{x}_{1} d \mathbf{p}_{1}}{(2 \pi)^{3}} \ldots \frac{d \mathbf{x}_{N} d \mathbf{p}_{N}}{(2 \pi)^{3}} \exp \left[-\frac{E\left(\mathbf{x}_{1}, \ldots, \mathbf{x}_{N} ; \mathbf{p}_{1}, \ldots, \mathbf{p}_{N}\right)}{T}\right],
$$

where $T$ is the system temperature, the particle degeneracy factor is assumed to be equal to 1 , and $E$ is the microscopic $N$-particle energy usually presented as the sum of potential and kinetic terms,

$$
E\left(\mathbf{x}_{1}, \ldots, \mathbf{x}_{N} ; \mathbf{p}_{1}, \ldots, \mathbf{p}_{N}\right)=U\left(\mathbf{x}_{1}, \ldots, \mathbf{x}_{N}\right)+\sum_{i=1}^{N} \frac{\mathbf{p}_{i}^{2}}{2 m}
$$

with $m$ being the particle mass. For the $N$-particle energy given by (3i) the integration over momentum in Eq. (2) can be done explicitly,

$$
Z_{c e}(V, T, N)=\frac{1}{N !}\left(\frac{m T}{2 \pi}\right)^{3 N / 2} \int_{V} d \mathbf{x}_{1} \ldots d \mathbf{x}_{N} \exp \left[-\frac{U\left(\mathbf{x}_{1}, \ldots, \mathbf{x}_{N}\right)}{T}\right] .
$$

\footnotetext{
${ }^{3}$ For several conserved charges $\left\{Q_{i}\right\}$ the number of possible ensembles is larger, as each charge can be treated either canonically or grand canonically.
} 
The particle coordinate $\mathbf{x}_{1}, \ldots, \mathbf{x}_{N}$ are integrated over the system volume $V$. The CE thermodynamical functions can be expressed in terms of the free energy,

$$
F(V, T, N)=-T \ln Z_{c e}(V, T, N)
$$

The CE pressure, entropy, chemical potential, and average energy are:

$$
p=-\left(\frac{\partial F}{\partial V}\right)_{T, N}, \quad S=-\left(\frac{\partial F}{\partial T}\right)_{V, N}, \quad \mu=\left(\frac{\partial F}{\partial N}\right)_{T, V}, \quad \bar{E}=F+T S .
$$

For the non-interacting particles, the potential energy vanishes, $U\left(\mathbf{x}_{1}, \ldots, \mathbf{x}_{N}\right)=0$, thus, the ideal gas free energy is:

$$
F_{i d}(V, T, N) \cong-N T-N T \ln \left[\frac{V}{N}\left(\frac{m T}{2 \pi}\right)^{3 / 2}\right]
$$

where we have assumed $N \gg 1$ and, thus, $\ln N ! \cong N \ln N-N$. The thermodynamical functions of the ideal gas from Eqs. (6],7) read:

$$
p=\frac{N T}{V}, \quad S=\frac{5}{2} N+N \ln \left[\frac{V}{N}\left(\frac{m T}{2 \pi}\right)^{3 / 2}\right], \quad \mu=-T \ln \left[\frac{V}{N}\left(\frac{m T}{2 \pi}\right)^{3 / 2}\right], \quad \bar{E}=\frac{3}{2} N T .
$$

The $(V, E, N)$ Micro-Canonical Ensemble (MCE) partition function of $N$ non-relativistic Boltzmann particles reads:

$$
Z_{m c e}(V, E, N)=\frac{1}{N !} \int \frac{d \mathbf{x}_{1} d \mathbf{p}_{1}}{(2 \pi)^{3}} \ldots \frac{d \mathbf{x}_{N} d \mathbf{p}_{N}}{(2 \pi)^{3}} \delta\left[E-U\left(\mathbf{x}_{1}, \ldots, \mathbf{x}_{N}\right)-\sum_{j=1}^{N} \frac{\mathbf{p}_{j}^{2}}{2 m}\right]
$$

The MCE entropy is defined as,

$$
S(V, E, N)=\ln \left[E_{0} Z_{m c e}(V, E, N)\right]
$$

where $E_{0}$ is an arbitrary constant with a dimension of energy. For non-interacting particles, $U\left(\mathbf{x}_{1}, \ldots, \mathbf{x}_{N}\right)=0$, one finds,

$$
Z_{m c e}(V, E, N)=\frac{V^{N}(E m / 2 \pi)^{3 N / 2}}{E N ! \Gamma(3 N / 2)}, \quad S(V, E, N) \cong \frac{5}{2} N+N \ln \left[\frac{V}{N}\left(\frac{m E}{3 N \pi}\right)^{3 / 2}\right] .
$$

It has been assumed that $N \gg 1$ and $E \gg E_{0}$, thus, $\Gamma(3 N / 2) \cong 3 N / 2[\ln (3 N / 2)-1]$, $N ! \cong N(\ln N-1)$, and $\ln \left(E_{0} \cdot E^{3 N / 2-1}\right) \cong \ln \left(E^{3 N / 2}\right)$.

The MCE temperature, pressure, and chemical potential are the following:

$$
\frac{1}{T}=\left(\frac{\partial S}{\partial E}\right)_{V, N}, \quad \frac{p}{T}=\left(\frac{\partial S}{\partial V}\right)_{E, N}, \quad \frac{\mu}{T}=-\left(\frac{\partial S}{\partial N}\right)_{V, E} .
$$


For non-interacting particles they read,

$$
\frac{1}{T}=\frac{3}{2} \frac{N}{E}, \quad \frac{p}{T}=\frac{N}{V}, \quad \frac{\mu}{T}=-\ln \left[\frac{V}{N}\left(\frac{m E}{3 N \pi}\right)^{3 / 2}\right] .
$$

If $\bar{E}=E$, the ideal gas system of $N$ particles in the volume $V$ has the same temperature, pressure, and entropy in the MCE (11, 13) and in the CE (8) . This means the thermodynamical equivalence of $\mathrm{CE}$ and $\mathrm{MCE}$ at $N \gg 1$.

\section{B. Pressure Canonical and Pressure Micro-Canonical Ensembles}

The particle coordinates and momenta for the considered system are denoted as $\mathbf{x}_{1}, \ldots, \mathbf{x}_{N}$ and $\mathbf{p}_{1}, \ldots, \mathbf{p}_{N}$, respectively. The $E$ given by Eq. (3) denotes the system energy, and $V$ is the system volume. The 'thermostat' is now introduced with corresponding particle coordinates, $\mathbf{X}_{1}, \ldots, \mathbf{X}_{N_{T}}$, and momenta, $\mathbf{P}_{1}, \ldots, \mathbf{P}_{N_{T}}$, and with $E_{T}$ and $V_{T}$ being the thermostat energy and volume, respectively. The system plus thermostat is described by the MCE, i.e. the total energy and the total volume are assumed to be fixed: $E+E_{T}=E^{*}=$ const , $V+V_{T}=V^{*}=$ const . The probability distribution of system particle coordinates and momenta is proportional to,

$$
f_{V}\left(\mathbf{x}_{1}, \ldots, \mathbf{x}_{N} ; \mathbf{p}_{1}, \ldots, \mathbf{p}_{N}\right) \propto \int \prod_{i=1}^{N_{T}} d^{3} \mathbf{X}_{i} d^{3} \mathbf{P}_{i} \delta\left(E+\sum_{j=1}^{N_{T}} \frac{\mathbf{P}_{j}^{2}}{2 M}-E^{*}\right)
$$

where the thermostat particle coordinates $\mathbf{X}_{1}, \ldots, \mathbf{X}_{N_{T}}$ are integrated over the thermostat volume $V_{T}$, and the system particle coordinates $\mathbf{x}_{1}, \ldots, \mathbf{x}_{N}$ are contained in the volume $V$. The particles in the thermostat are assumed to be non-interacting. Thus, $E_{T}=\sum_{j=1}^{N_{T}} \mathbf{P}_{j}^{2} /(2 M)$, with $M$ being the particle mass. The momentum integration in Eq. (14) gives,

$$
\begin{aligned}
& \int d^{3} \mathbf{P}_{1} \ldots d^{3} \mathbf{P}_{N_{T}} \delta\left(E+\sum_{j=1}^{N_{T}} \frac{\mathbf{P}_{j}^{2}}{2 M}-E^{*}\right) \propto\left(E^{*}-E\right)^{3 N_{T} / 2-1} \propto\left(1-\frac{E}{E^{*}}\right)^{3 N_{T} / 2-1} \\
& \cong\left(1-\frac{E}{E_{T}}\right)^{3 N_{T} / 2-1} \cong\left(1-\frac{E}{3 N_{T} T / 2}\right)^{3 N_{T} / 2} \cong \exp (-E / T)
\end{aligned}
$$

where it has been assumed, $N_{T} \rightarrow \infty, E / E_{T} \rightarrow 0$, and the ideal gas equation for the thermostat energy, $E_{T}=3 T N_{T} / 2$, has been used. The integration over thermostat particle coordinates $\mathbf{X}_{i}$ 
in Eq. (14) gives the factor depending on the system volume $V$,

$$
\begin{aligned}
\int_{V_{T}} d^{3} \mathbf{X}_{1} \ldots d^{3} \mathbf{X}_{N_{T}} & =V_{T}^{N_{T}}=\left(V^{*}-V\right)^{N_{T}} \propto\left(1-\frac{V}{V^{*}}\right)^{N_{T}} \cong\left(1-\frac{V}{V_{T}}\right)^{N_{T}} \\
& =\left(1-\frac{V}{\left(T N_{T} / p_{0}\right)}\right)^{N_{T}} \cong \exp \left(-\frac{p_{0} V}{T}\right),
\end{aligned}
$$

where it has been assumed, $V_{T} \rightarrow \infty, N_{T} \rightarrow \infty, V / V_{T} \rightarrow 0$, and the ideal gas equation for the thermostat pressure, $p_{0}=T N_{T} / V_{T}$, has been used.

One obtains from Eqs. (14 16),

$$
f_{V}\left(\mathbf{x}_{1}, \ldots, \mathbf{x}_{N}, \mathbf{p}_{1}, \ldots, \mathbf{p}_{N}\right)=\frac{1}{Z_{p c e}\left(p_{0}, T, N\right)} \frac{1}{N !} \exp \left(-\frac{p_{0} V+E}{T}\right),
$$

where the system energy $E$ in (17) depends on particle coordinates and momenta according to Eq. (3). The function $Z_{p c e}\left(p_{0}, T, N\right)$ in Eq. (17) is the $\left(p_{0}, T, N\right)$ Pressure Canonical Ensemble (PCE) partition function. It is defined by the normalization condition,

$$
\int_{0}^{\infty} d V \int \prod_{i=1}^{N}\left[\frac{d^{3} \mathbf{x}_{i} d^{3} \mathbf{p}_{i}}{(2 \pi)^{3}}\right] f_{V}\left(\mathbf{x}_{1}, \ldots, \mathbf{x}_{N} ; \mathbf{p}_{1}, \ldots, \mathbf{p}_{N}\right)=1,
$$

and it equals to,

$$
Z_{p c e}\left(p_{0}, T, N\right)=\int_{0}^{\infty} d V \exp \left(-\frac{p_{0} V}{T}\right) Z_{c e}(V, T, N),
$$

where $Z_{c e}(V, T, N)$ is the CE partition function given by Eq. (22).

Using $Z_{c e}=\exp (-F / T)$, the probability volume distribution in the PCE can be presented as:

$$
W_{p c e}(V)=\frac{1}{Z_{p c e}\left(p_{0}, T, N\right)} \exp \left[-\frac{F(V, T, N)+p_{0} V}{T}\right] .
$$

First, one finds the maximum of $W_{\text {pce }}(V)$, which defines the most probable value of the volume, $V=V_{0}$,

$$
\left[\frac{\partial F(V, T, N)}{\partial V}\right]_{V=V_{0}}+p_{0}=0 .
$$

By definition, $-\partial F / \partial V$ is the CE pressure (8). Thus, the PCE equation of state (21) has clear physical meaning: the internal pressure $p$ for the most probable volume $V_{0}$ equals to the fixed external pressure $p_{0}$,

$$
p\left(V_{0}, N, T\right)=p_{0} .
$$


In the TL the volume distribution (20) can be approximated as,

$$
\begin{aligned}
& W_{p c e}(V) \cong \frac{1}{Z_{p c e}\left(p_{0}, T, N\right)} \exp \left[-\frac{F\left(V_{0}, T, N\right)+p_{0} V_{0}}{T}\right] \\
& \times \exp \left[-\frac{1}{2 T}\left(\frac{\partial^{2} F(V, T, N)}{\partial V^{2}}\right)_{V=V_{0}}\left(V-V_{0}\right)^{2}\right] \equiv\left(2 \pi \omega_{V} V_{0}\right)^{-1 / 2} \exp \left[-\frac{\left(V-V_{0}\right)^{2}}{2 \omega_{V} V_{0}}\right] .
\end{aligned}
$$

Thus, the most probable volume, $V_{0}$, and the average volume $\bar{V}$ are equal to each other in the TL,

$$
\bar{V} \equiv \int_{0}^{\infty} d V V W_{p c e}(V) \cong V_{0}
$$

and $\omega_{V}$ introduced in Eq. (23) defines the scaled variance of the volume fluctuations,

$$
\omega_{V} \equiv \frac{\overline{V^{2}}-\bar{V}^{2}}{\bar{V}}=\frac{T}{V_{0}}\left[\frac{\partial^{2} F(V, T, N)}{\partial V^{2}}\right]_{V=V_{0}}^{-1}=-\frac{T}{V_{0}}\left[\frac{\partial p(V, T, N)}{\partial V}\right]_{V=V_{0}}^{-1} .
$$

For the ideal gas pressure (8) one gets:

$$
\bar{V} \cong V_{0}=\frac{N T}{p_{0}}, \quad \omega_{V} \cong \frac{T}{p_{0}} .
$$

The Eqs. (23) 25$)$ are valid if $(\partial p / \partial V)$ is negative. This is the case for the 'normal' equation of state, but is not valid for either a 1st order phase transition or critical point. Note that the TL $N, V_{0} \rightarrow \infty$, with $N / V_{0}$ being finite, is assumed to make clear notions of the phase transition or critical point.

1st Order Phase Transition. In the casei of the 1st order phase transition $\partial p / \partial V=0$ [19] for $V_{1}<V<V_{2}$ in the mixed phase. According to Eq. (20) this leads to $W_{p c e}(V)=$ const $\cong$ $\left(V_{2}-V_{1}\right)^{-1}$ for $V_{1}<V<V_{2}$. Introducing the notation, $V_{2}=\gamma V_{1}$, with $\gamma=$ const $>1$, one finds at $V_{1} \rightarrow \infty$,

$$
\begin{aligned}
& \bar{V} \cong \int_{V_{1}}^{V_{2}} V W_{p c e}(V) d V=\frac{\gamma+1}{2} V_{1} \\
& \omega_{V} \cong \frac{1}{\bar{V}}\left[\int_{V_{1}}^{V_{2}} V^{2} W_{p c e}(V) d V-\bar{V}^{2}\right]=\frac{1}{3}\left(\frac{\gamma-1}{\gamma+1}\right)^{2} \bar{V} .
\end{aligned}
$$

Thus, the volume fluctuations are anomalously large, $\omega_{V} \propto \bar{V} \rightarrow \infty$.

Critical Point. At the critical point it follows [19],

$$
\frac{\partial p}{\partial V}=\frac{\partial^{2} p}{\partial V^{2}}=0
$$


In this case, Eq. (20) takes the form,

$$
\begin{aligned}
& W_{p c e}(V) \cong \frac{1}{Z_{p c e}\left(p_{0}, T, N\right)} \exp \left[-\frac{F\left(V_{0}, T, N\right)+p_{0} V_{0}}{T}\right] \\
& \times \exp \left[-\frac{1}{T} \frac{1}{4 !}\left(\frac{\partial^{4} F(V, T, N)}{\partial V^{4}}\right)_{V=V_{0}}\left(V-V_{0}\right)^{4}\right] \equiv \frac{2 A_{0}^{1 / 4}}{\Gamma(1 / 4)} \exp \left[-A_{0}\left(V-V_{0}\right)^{4}\right],
\end{aligned}
$$

where $A_{0} \equiv-(24 T)^{-1}\left(\partial^{3} p / \partial V^{3}\right)_{V=V_{0}}$. The volume distribution (30) leads to the scaled variance for the volume fluctuations,

$$
\omega_{V}=\frac{\overline{V^{2}}-\bar{V}^{2}}{\bar{V}} \cong \frac{1}{V_{0}} \int_{0}^{\infty} d V\left(V-V_{0}\right)^{2} W_{p c e}(V)=\frac{1}{V_{0}} \frac{\Gamma(3 / 4)}{\Gamma(1 / 4)} A_{0}^{-1 / 2}
$$

where the Gamma functions in Eqs. (30) are $\Gamma(1 / 4) \cong 3.626$ and $\Gamma(3 / 4) \cong 1.225$. Finally, one finds for (31),

$$
\omega_{V} \cong \frac{1.656 T^{1 / 2}}{V_{0}}\left[-\left(\frac{\partial^{3} p}{\partial V^{3}}\right)_{V=V_{0}}\right]^{-1 / 2}
$$

In order to estimate the scaled variance (32) of the volume fluctuations at the critical point we use the van der Waals (VdW) equation of state [19],

$$
\left(p+a \frac{N^{2}}{V^{2}}\right)(V-b N)=N T .
$$

The critical point is defined by the following equations

$$
\begin{aligned}
\frac{\partial p}{\partial V} & =-\frac{N T}{(V-N b)^{2}}+\frac{2 N^{2} a}{V^{3}}=0, \\
\frac{\partial^{2} p}{\partial V^{2}} & =\frac{2 N T}{(V-N b)^{3}}-\frac{6 N^{2} a}{V^{4}}=0 .
\end{aligned}
$$

They give,

$$
T_{c r}=\frac{8}{27} \frac{a}{b}, \quad V_{c r}=3 N b, \quad p_{c r}=\frac{1}{27} \frac{a}{b^{2}} .
$$

At the critical point one finds,

$$
\left(\frac{\partial^{3} p}{\partial V^{3}}\right)_{c r}=\left[-\frac{6 N T}{(V-N b)^{4}}+\frac{24 N^{2} a}{V^{5}}\right]_{c r}=-\frac{a}{9^{2} b^{5}} \frac{1}{N^{3}} .
$$

Thus, for $T=T_{c r}, p_{0}=p_{c r}$, and $\bar{V} \cong V_{0}=V_{c r}$, and Eqs. (32/37) give,

$$
\omega_{V} \cong \frac{1.656 T^{1 / 2}}{V_{c r}}\left[-\left(\frac{\partial^{3} p}{\partial V^{3}}\right)_{V=V_{c r}}\right]^{-1 / 2} \cong 2.703 b \sqrt{N}
$$


The volume fluctuations at the critical point are anomalously large, $\omega_{V} \propto N^{1 / 2} \propto \bar{V}^{1 / 2} \rightarrow \infty$.

Similarly to the PCE we introduce now the $\left(s_{0}, E, N\right)$ Pressure Micro-Canonical ensemble $(\mathrm{PMC})$

$$
Z_{p m c}\left(s_{0}, E, N\right)=\int_{0}^{\infty} d V \exp \left(-s_{0} V\right) Z_{m c e}(V, E, N)
$$

where the parameter $s_{0}=p_{0} / T_{0}$ is defined by the external conditions of the thermostat pressure $p_{0}$ and the thermostat temperature $T_{0}$. The micro-canonical energy $E$ is assumed to be fixed. Thus, there is no energy exchange between the 'thermostat' and considered system, and the internal MCE temperature $T$ (12) may differ from $T_{0}$. Using Eq. (10), the volume distribution in the PMC can be presented in the form,

$$
\begin{aligned}
W_{p m c}(V) & =\frac{1}{Z_{p m c}\left(s_{0}, E, N\right)} \exp \left[-s_{0} V+S(V, E, N)\right] \\
& \cong \frac{1}{Z_{p m c}\left(s_{0}, E, N\right)} \exp \left[-s_{0} V_{0}+S\left(V_{0}, E, N\right)+\frac{1}{2}\left(\frac{\partial^{2} S}{\partial V^{2}}\right)_{V=V_{0}}\left(V-V_{0}\right)^{2}\right]
\end{aligned}
$$

The most probable (and average) volume $V_{0}$ is defined by the condition,

$$
-s_{0}+\left(\frac{\partial S(V, E, N)}{\partial V}\right)_{V=V_{0}}=0
$$

The Eq. (41) corresponds to,

$$
\frac{p}{T}=\frac{p_{0}}{T_{0}}
$$

which is similar to Eq. (22) in the PCE. The scaled variance of the volume fluctuations $\omega_{V}$ in the PMC equals to:

$$
\omega_{V} \cong-\left[V_{0}\left(\partial^{2} S / \partial V^{2}\right)_{V=V_{0}}\right]^{-1}=-\frac{T}{V_{0}}\left[\frac{\partial p(V, E, N)}{\partial V}\right]_{V=V_{0}}^{-1}
$$

which is again similar to Eq. (25) in the PCE. Thus, the average volume $V_{0}$ and scaled variance of the volume fluctuations $\omega_{V}$ are identical in the PCE and PMC in the TL. For the ideal gas one finds,

$$
\langle V\rangle_{p m c} \cong V_{0}=\frac{N}{s_{0}}, \quad \omega_{V}=\frac{1}{s_{0}}
$$

which coincide with (26) in the PCE. 


\section{Grand Canonical Ensemble}

The $(V, T, \mu)$ Grand Canonical Ensemble (GCE) partition function of non-relativistic noninteracting Boltzmann particles reads:

$$
Z_{g c e}(V, T, \mu)=\sum_{N=0}^{\infty} \exp \left(\frac{\mu N}{T}\right) Z_{c e}(V, T, N)
$$

where $Z_{c e}(V, T, N)$ is given by Eq. (2) and $\mu$ is the chemical potential. The GCE pressure is calculated as,

$$
p(T, \mu)=\frac{T}{V} \ln \left[Z_{g c e}(V, T, \mu)\right]
$$

and it does not depend on the volume $V$ in the TL. The CE, MCE, and GCE are thermodynamically equivalent at $V \rightarrow \infty$. Note that the GCE is the most convenient one from the technical point of view.

There is, however, an evident problem in the formulation of the $\left(p_{0}, T, \mu\right)$ Pressure Grand Canonical ensemble (PGC). The PGC partition function is obtained by extending Eq. (19),

$$
Z_{p g c}\left(p_{0}, T, \mu\right)=\int_{0}^{\infty} d V \exp \left(-\frac{p_{0} V}{T}\right) Z_{g c e}(V, T, \mu)=\frac{T}{p_{0}-p(T, \mu)}
$$

where $p(T, \mu)$ is the GCE pressure (46). The $\left(p_{0}, T, \mu\right)$-ensemble has a unique property. Among 8 possible ensembles this is the only one where the system description includes only intensive quantites, $p_{0}, T$ and $\mu$. For $p(T, \mu)=p_{0}$, the system volume is undefined. In the domain $p(T, \mu) \geq p_{0}$, the PGC partition function does not exist as the integral over the volume in Eq. (47) diverges. For $p(T, \mu)<p_{0}$, the volume distribution has the form:

$$
W_{p g c}(V)=\frac{1}{Z_{p g c}\left(p_{0}, T, \mu\right)} \exp \left[-V\left(\frac{p_{0}-p}{T}\right)\right]
$$

The most probable volume $V_{0}$ equals to zero, but the average volume is:

$$
\bar{V}=\int_{0}^{\infty} d V V W_{p g c}(V)=\frac{T}{p_{0}-p(T, \mu)} .
$$

This special ensemble will be discussed further in details for the ultra-relativistic gas in the next section. 


\section{ULTRA-RELATIVISTIC GAS}

In this section several examples of the pressure ensembles for the ultra-relativistic $(m=0)$ ideal gas of Boltzmann particles are considered. For simplicity only statistical systems without conserved charges are discussed. Thus, the number of particles is not restricted and chemical potential equals to zero. Furthermore, the Boltzmann statistics is used and the degeneracy factor is assumed to be one.

\section{A. Grand Canonical and Grand Micro-Canonical Ensembles}

The $(V, T) \mathrm{GCE}^{4}$ partition function of massless non-interacting neutral Boltzmann particles reads:

$$
Z_{\text {gce }}(V, T)=\sum_{N=0}^{\infty} \frac{1}{N !}\left(\frac{V}{2 \pi^{2}}\right)^{N} \int_{0}^{\infty} \prod_{i=1}^{N} p_{i}^{2} d p_{i} \exp \left(-\frac{p_{i}}{T}\right)=\sum_{N=0}^{\infty} \frac{1}{N !}\left(\frac{V T^{3}}{\pi^{2}}\right)^{N}=\exp (\bar{N})
$$

where $\bar{N} \equiv\langle N\rangle_{g c e}=V T^{3} / \pi^{2}$ is the GCE average number of particles. The GCE system pressure and average energy are:

$$
\begin{aligned}
p & =\frac{T}{V} \ln Z_{\text {gce }}=\frac{T^{4}}{\pi^{2}}=T n(T), \\
\langle E\rangle_{\text {gce }} & \equiv \bar{E}=T^{2} \frac{\partial \ln Z_{\text {gce }}}{\partial T}=\frac{3}{\pi^{2}} V T^{4}=\varepsilon(T) V,
\end{aligned}
$$

where $n(T)=\bar{N} / V=T^{3} / \pi^{2}$ and $\varepsilon(T)=3 T^{4} / \pi^{2}$ are the particle number density and energy density, respectively. The GCE multiplicity distribution has the Poisson form,

$$
P_{g c e}(N ; V, T)=\frac{\bar{N}^{N}}{N !} \exp (-\bar{N})
$$

and the scaled variance of particle number distribution (53) equals to:

$$
\omega_{g c e} \equiv \frac{\left\langle N^{2}\right\rangle_{g c e}-\langle N\rangle_{g c e}^{2}}{\langle N\rangle_{g c e}}=1
$$

\footnotetext{
4 The chemical potential connected to the number of particles equals to zero.
} 
The $(V, E)$ Grand Micro-Canonical ensemble ${ }^{5}$ (GMC) partition function is [8]]:

$$
\begin{aligned}
Z_{g m c}(V, E) & =\sum_{N=1}^{\infty} \frac{1}{N !}\left(\frac{V}{2 \pi^{2}}\right)^{N} \int_{0}^{\infty} \prod_{i=1}^{N} p_{i}^{2} d p_{i} \delta\left(E-\sum_{j=1}^{N} p_{j}\right) \\
& \equiv \sum_{N=1}^{\infty} W_{N}(V, E)=\frac{1}{E} \sum_{N=1}^{\infty} \frac{A^{N}}{N !(3 N-1) !}=\frac{A}{2 E}{ }_{0} F_{3}\left(; \frac{4}{3}, \frac{5}{3}, 2 ; \frac{A}{27}\right)
\end{aligned}
$$

where ${ }_{0} F_{3}$ is the generalized hyper-geometric function [21], and

$$
A \equiv \frac{V E^{3}}{\pi^{2}} .
$$

The GMC particle number distribution function equals to:

$$
P_{g m c}(N ; V, E) \equiv \frac{W_{N}(V, E)}{Z_{g m c}(V, E)}=\frac{1}{Z_{g m c}(V, E)} \frac{A^{N}}{E N !(3 N-1) !} .
$$

It is defined for $N \geq 1$. The average number of particles in the GMC equals to [8]:

$$
\langle N\rangle_{g m c} \cong\left(\frac{A}{27}\right)^{1 / 4}
$$

In the large volume limit the mean multiplicities in the GME and the GCE are equal, $\langle N\rangle_{g m c}=$ $\bar{N}$ providing $E=\bar{E}$ and the GMC and the GCE volumes are equal. The temperature and the pressure in the GME are equal to:

$$
T=\frac{E}{3 \bar{N}}=\left(\frac{\pi^{2} E}{3 V}\right)^{1 / 4}, \quad p=\frac{\bar{N} T}{V}=\frac{T^{4}}{\pi^{2}},
$$

and they coincide with the corresponding quantities (151/52) in the GCE.

For $\bar{N} \gg 1$ the particle number distribution in the GME (57) can be approximated by the Gaussian one:

$$
P_{g m c}(N ; V, E) \cong\left(2 \pi \omega_{g c e} \bar{N}\right)^{-1 / 2} \exp \left[-\frac{(N-\bar{N})^{2}}{2 \omega_{g m c} \bar{N}}\right]
$$

with the GME scaled variance

$$
\omega_{g m c}=\frac{\left\langle N^{2}\right\rangle_{g m c}-\langle N\rangle_{g m c}^{2}}{\langle N\rangle_{g m c}}=\frac{1}{4} .
$$

The Poisson distribution $P_{g c e}(N)$ (53) for $\bar{N} \gg 1$ can be also approximated by the Gauss distribution (60), but with $\omega_{\text {gce }}=1$. In the TL the particle number distributions in the GME and GCE have both the Gauss form [12]. The average number of particles is the same, $\langle N\rangle_{g m c} \cong \bar{N}$, but the scaled variance is different, $\omega_{g c e}=1$ and $\omega_{g m c}=1 / 4$.

\footnotetext{
${ }^{5}$ The energy is fixed, but the number of particles is not. The chemical potential connected to the number of particles equals to zero. Thus, this ensemble is named the Grand Micro-Canonical Ensemble [9].
} 


\section{B. Pressure Grand Canonical Ensemble}

The $\left(p_{0}, T\right)$ Pressure Grand Canonical ensemble (PGC) is defined as the ensemble with the fixed external pressure $p_{0}$, temperature $T$, and the chemical potential connected to the number of particles equals to zero. The PGC partition function equal to [20]:

$$
Z_{p g c}\left(p_{0}, T\right) \equiv \int_{0}^{\infty} d V \exp \left(-\frac{p_{0} V}{T}\right) Z_{g c e}(V, T)=\frac{T}{p_{0}-p(T)},
$$

where the relation (51) between $p(T)$ and $Z_{g c e}$ has been used. The value of $p_{0}$ has a physical meaning of the external pressure. A convergence of the integral over the volume in Eq. (62) requires the inequality,

$$
p(T)=\frac{1}{\pi^{2}} T^{4}<p_{0} .
$$

Thus, at each fixed value of $p_{0}$, there is a 'limiting temperature' $T^{*}$ in the PGC:

$$
T<T^{*}=\left(\pi^{2} p_{0}\right)^{1 / 4}
$$

The probability volume distribution in the $\mathrm{PGC}$ is:

$$
W_{p g c}(V)=\frac{1}{Z_{p g c}\left(p_{0}, T\right)} \exp \left[-V\left(\frac{p_{0}-p(T)}{T}\right)\right] .
$$

It gives the most probable volume equal to zero, $V_{0}=0$, and the average value,

$$
\langle V\rangle_{p g c} \equiv \bar{V}=\int_{0}^{\infty} d V V W_{p g c}(V)=-T \frac{\partial \ln Z_{p g c}\left(p_{0}, T\right)}{\partial p_{0}}=\frac{T}{p_{0}-p(T)},
$$

which is not equal to zero and may even go to infinity at $T \rightarrow T^{*}$. Using Eq. (66), the volume distribution (65) can be written as,

$$
W_{p g c}(V)=\bar{V}^{-1} \exp (-V / \bar{V})
$$

For the mean values of energy and particle number in the PGC one finds:

$$
\begin{aligned}
\langle E\rangle_{p g c} & =\int_{0}^{\infty} d V W_{p g c}(V)\langle E\rangle_{g c e}=\frac{3}{\pi^{2}} T^{4} \bar{V}=\varepsilon(T) \bar{V}, \\
\langle N\rangle_{p g c} & =\int_{0}^{\infty} d V W_{p g c}(V)\langle N\rangle_{g c e}=\frac{1}{\pi^{2}} T^{3} \bar{V}=n(T) \bar{V},
\end{aligned}
$$

where $n(T)$ and $\varepsilon(T)$ are the particle number density and energy density of the GCE given by Eq. (51) and Eq. (52), respectively. 
The independent variables are $(V, T)$ in the GCE and $\left(p_{0}, T\right)$ in the PGC. If the GCE volume is chosen to be equal to the average volume of the PGC, then Eqs. (68) and (69) give: $\langle E\rangle_{p g c}=\bar{E}$ and $\langle N\rangle_{p g c}=\bar{N}$, i.e. the GCE and PGC are thermodynamically equivalent. The fluctuations of $E$ and $N$ are however different in these two ensembles. Calculating,

$$
\left\langle V^{2}\right\rangle_{p g c}=\int_{0}^{\infty} d V V^{2} W_{p g c}(V)=\frac{T^{2}}{Z_{p g c}} \frac{\partial^{2} Z_{p g c}}{\partial p_{0}^{2}}=2\left[\frac{T}{p_{0}-p(T)}\right]^{2}=2 \bar{V}^{2},
$$

one finds,

$$
\omega_{V}=\frac{\left\langle V^{2}\right\rangle_{p g c}-\langle V\rangle_{p g c}^{2}}{\langle V\rangle_{p g c}}=\bar{V}
$$

Thus, in the TL limit $\bar{V} \rightarrow \infty$ the volume fluctuations become anomalously large. This, in turn, leads to anomalous energy and particle number fluctuations. The particle number distribution in the PGC has the form of the geometrical distribution:

$$
P_{p g c}\left(N ; p_{0}, T\right)=\int_{0}^{\infty} d V W_{p g c}(V) P_{g c e}(N, V, T)=(1-\eta) \eta^{N},
$$

where $P_{\text {gce }}(N, V, T)$ has been taken from Eq. (53), and $\eta \equiv\left(T / T^{*}\right)^{4}<1$. The most probable number of particles is $N=0$, whereas the average value (69) is larger than zero. It equals to $\langle N\rangle_{p g c}=\eta(1-\eta)^{-1}$ and may even go to infinity at $\eta \rightarrow 1$. This happens if $T \rightarrow T^{*}$. From Eq. (72) it follows:

$$
\omega_{p g c}=\frac{\left\langle N^{2}\right\rangle_{p g c}-\langle N\rangle_{p g c}^{2}}{\langle N\rangle_{p g c}}=1+\frac{\eta}{1-\eta}=1+n(T) \bar{V} .
$$

The first term in the r.h.s. of Eq. (73) corresponds to the Poisson fluctuations (54) of the GCE at fixed volume, whereas the second term comes from the volume fluctuations at fixed particle number density. The multiplicity distribution (72) can be rewritten as,

$$
P_{p g c}\left(N ; p_{0}, T\right) \equiv P_{p g c}(N ; \bar{N})=\frac{1}{\bar{N}+1} \exp \left[-N \ln \left(1+\frac{1}{\bar{N}}\right)\right] .
$$

For $\bar{N} \gg 1$ the distribution $P_{p g c}$ approches:

$$
P_{p g c}(N ; \bar{N}) \cong \frac{1}{\bar{N}} \exp \left(-\frac{N}{\bar{N}}\right)
$$

The particle number distribution $P_{p g c}(N)$ (75) satisfies the so called KNO-scaling [18]. 


\section{Pressure Grand Micro-Canonical Ensemble}

The $\left(s_{0}, E\right)$ Pressure Grand Micro-canonical ensemble (PGM) is defined as the statistical ensemble with fixed energy $E$ and fixed external parameter $s_{0}=p_{0} / T_{0}$. As before, the chemical potential connected to the number of particles equals to zero. The PGM partition function is equal to:

$$
\begin{aligned}
& Z_{\text {pgm }}\left(s_{0}, E\right) \equiv \int_{0}^{\infty} d V \exp \left(-s_{0} V\right) Z_{g m c}(V, E) \\
& =\frac{1}{E} \sum_{N=1}^{\infty}\left(\frac{E^{3}}{\pi^{2}}\right)^{N} \frac{1}{N !(3 N-1) !} \int_{0}^{\infty} d V \exp \left(-s_{0} V\right) V^{N}=\frac{1}{s_{0} E} \sum_{N=1}^{\infty} \frac{B^{N}}{(3 N-1) !},
\end{aligned}
$$

where

$$
B \equiv \frac{E^{3}}{s_{0} \pi^{2}}
$$

Using Eq. [22],

$$
\sum_{k=0}^{\infty} \frac{x^{3 k}}{(3 k) !}=\frac{1}{3}\left[\exp (x)+2 \exp (-x / 2) \cos \left(\frac{\sqrt{3}}{2} x\right)\right],
$$

one finds at $B \gg 1$,

$$
\ln \left[Z_{p g m}\left(s_{0}, E\right)\right] \cong B^{1 / 3}
$$

In the TL, the volume distribution in the PGM can be found from (76) using the asymptotic behavior of ${ }_{0} F_{3}$ function [21],

$$
\ln \left[Z_{g m c}(V, E)\right]=\ln \left[\frac{A}{2 E}{ }_{0} F_{3}\left(; \frac{4}{3}, \frac{5}{3}, 2 ; \frac{A}{27}\right)\right] \cong 4\left(\frac{V E^{3}}{27 \pi^{2}}\right)^{1 / 4} .
$$

The volume distribution in the PGM is then proportional to,

$$
\begin{aligned}
W_{p g m}(V) & \propto \exp \left[4\left(\frac{V E^{3}}{27 \pi^{2}}\right)^{1 / 4}-s_{0} V\right] \equiv \exp [\phi(V)] \\
& \cong \exp \left[\phi\left(V_{0}\right)+\frac{1}{2}\left(\frac{\partial^{2} \phi}{\partial V^{2}}\right)_{V=V_{0}}\left(V-V_{0}\right)^{2}\right]
\end{aligned}
$$

The most probable volume $V_{0}$ in the PGM is defined by the condition,

$$
\left(\frac{\partial \phi}{\partial V}\right)_{V=V_{0}}=\left(\frac{E^{3}}{27 \pi^{2} V_{0}^{3}}\right)^{1 / 4}-s_{0}=0 .
$$


This gives,

$$
V_{0}=\frac{E}{3 \pi^{2 / 3} s_{0}^{4 / 3}} .
$$

One also finds,

$$
\left(\frac{\partial^{2} \phi}{\partial V^{2}}\right)_{V=V_{0}}=-\frac{3 s_{0}}{4 V_{0}} .
$$

Thus, the volume distribution in the PGM can be approximated as:

$$
W_{p g m}(V) \cong\left(2 \pi \omega_{V} V_{0}\right)^{-1 / 2} \exp \left[-\frac{\left(V-V_{0}\right)^{2}}{2 \omega_{V} V_{0}}\right],
$$

where $\omega_{V}=4 /\left(3 s_{0}\right)$ is the scaled variance of the volume fluctuations in the PGM. The average volume and its fluctuations in the PGM can be also calculated in terms of the partition function $Z_{p g m}\left(s_{0}, E\right)$ using Eqs. (76-79),

$$
\begin{aligned}
\langle V\rangle_{p g m} & =\int_{0}^{\infty} d V W_{p g m}(V) V=-\frac{\partial \ln Z_{p g m}}{\partial s_{0}} \cong \frac{E}{3 \pi^{2 / 3} s_{0}^{4 / 3}}, \\
\left\langle V^{2}\right\rangle_{p g m} & =\int_{0}^{\infty} d V W_{p g m}(V) V^{2}=\frac{1}{Z_{p g m}} \frac{\partial^{2} Z_{p g m}}{\partial s_{0}^{2}} \cong \frac{E^{2}}{9 \pi^{4 / 3} s_{0}^{8 / 3}}, \\
\omega_{V} & =\frac{\left\langle V^{2}\right\rangle_{p g m}-\langle V\rangle_{p g m}^{2}}{\langle V\rangle_{p g m}} \cong \frac{4}{3 s_{0}} .
\end{aligned}
$$

The condition (83) can be written as,

$$
s_{0}=\frac{1}{\pi^{2}} T^{3}=\frac{p}{T},
$$

and it means that the internal pressure $p$ equals to $s_{0} T$. The most probable volume $V_{0}$ (83) and average volume $\langle V\rangle_{p g m}($ (86) $)$ are then equal to each other, and both are equal to $E \pi^{2} /\left(3 T^{4}\right)$. This corresponds to the fixed volume in the MCE with fixed energy $E$ and the MCE temperature $T$. The volume distribution in the PGM is therefore different from that in the PGC (67). In contrast to the $\mathrm{PGC}$, there is an extensive variable, the energy $E$, in the PMG. This leads to the system average volume proportional to the energy and given by Eq. (83). The internal pressure equals to $p=s_{0} T$ (89). As a result, the volume fluctuations in the PGM are Gaussian (85) with the finite scaled variance (88). Using Eq. (89) the scaled variance (88) of the volume fluctuations can be expressed in terms of the MCE temperature, $\omega_{V}=4 \pi^{2} /\left(3 T^{3}\right)$. One also 
finds:

$$
\begin{aligned}
\langle N\rangle_{p g m} & =\frac{1}{Z_{p g m}} B \frac{\partial Z_{p g m}}{\partial B} \cong \frac{1}{3} B^{1 / 3}, \\
\left\langle N^{2}\right\rangle_{p g m} & =\frac{1}{Z_{p g m}} B \frac{\partial}{\partial B} B \frac{\partial Z_{p g m}}{\partial B} \cong \frac{1}{9} B^{1 / 3}+\frac{1}{9} B^{2 / 3}, \\
\omega_{p g m} & =\frac{\left\langle N^{2}\right\rangle_{p g m}-\langle N\rangle_{p g m}^{2}}{\langle N\rangle_{p g m}} \cong \frac{1}{3} .
\end{aligned}
$$

In the TL, the multiplicity distribution in the PGM can be approximated as:

$$
P_{p g m}\left(N ; s_{0}, E\right) \cong\left(2 \pi \omega_{p g m} \bar{N}\right)^{-1 / 2} \exp \left[-\frac{(N-\bar{N})^{2}}{2 \omega_{p g m} \bar{N}}\right],
$$

where $\bar{N}=B^{1 / 3} / 3 \cong\langle N\rangle_{p g m}$ and $\omega_{p g m}=1 / 3$. The scaled variance in the PGM can be presented in the TL as the following,

$$
\omega_{\text {pgm }}=\omega_{m c e}+\frac{1}{16} \omega_{V} n=\frac{1}{4}+\frac{1}{12}=\frac{1}{3},
$$

where $n=T^{3} / \pi^{2}$ is the MCE particle number density. The first term in the r.h.s. of Eq. (94), $\omega_{m c e}=1 / 4$, is due to the particle number fluctuations in the MCE with fixed volume, and the second term is the contribution due to the volume fluctuations.

\section{ENSEMBLES WITH EXTERNAL VOLUME FLUCTUATIONS}

The multiplicity distributions $P(N)$ in relativistic gases [7, 8, 9, 10, 11, 12, 13] are sensitive to conservation laws obeyed by the system, and therefore to fluctuations of extensive quantities $E$ and $Q$. The examples considered in the previous section demonstrate that the volume fluctuations also influence the particle number fluctuations. Thus, for the calculation of multiplicity distributions, the choice of the statistical ensemble is then not a matter of convenience, but a physical question. On the other hand, the fluctuations of extensive quantities $\vec{A} \equiv(V, E, Q)$ depend not on the system's physical properties, but rather on external conditions. One can imagine a huge variety of these conditions, thus, 8 statistical ensembles discussed in the previous sections are only some special examples.

A more general concept of the statistical ensembles based on Eq. (1) was recently suggested in Ref. [16]. The system volume may exhibit fluctuations described by the externally given distribution. When $V$ is the only fluctuating variable, Eq. (1) is reduced to

$$
P_{\alpha}(O)=\int d V P_{\alpha}(E, V, Q) P_{m c e}(O ; E, V, Q),
$$


where $P_{\alpha}(V)$ is externally given volume distribution.

The effect of volume fluctuations on the particle number fluctuations is calculated for the system of non-interacting massless Boltzmann particles with zero chemical potential. At fixed volume the system is treated within the GMC, and the particle number distribution is $P_{g m c}(N ; V, E)$ (157).

In the first example, the volume distribution is assumed to be:

$$
P_{\alpha}(V)=\left(2 \pi \omega_{V} a_{V}^{2} \bar{V}\right)^{-1 / 2} \exp \left[-\frac{(V-\bar{V})^{2}}{2 \omega_{V} a_{V}^{2} \bar{V}}\right],
$$

where $\omega_{V}=4 \pi^{2} /\left(3 T^{3}\right)$ coincides with (8ㅏ) in the PGM. The choice of $P_{\alpha}(V)$ results in a simple correspondence to the GMC and PGM in the TL. In Eq. (96), $a_{V}$ is a dimensionless tuneable parameter which determines the width of the distribution. In the limit $a_{V} \rightarrow 0$, Eq. (96) becomes a Dirac $\delta$-function, $\delta(V-\bar{V})$. This corresponds to the GMC. For $a_{V}=1$, Eq. (96) results in the PGM volume fluctuations (85) in the TL. The particle number distribution reads,

$$
P_{\alpha}(N)=\int_{0}^{\infty} d V P_{\alpha}(V) P_{g m c}(N ; V, E) .
$$

A substitution of $P_{\alpha}(V)$ in Eq. (97) by the distribution (96) results in $P_{\alpha}(N)$ in the TL given by the Gaussian,

$$
P_{\alpha}(N) \cong\left(2 \pi \omega_{\alpha} \bar{N}\right)^{-1 / 2} \exp \left[-\frac{(N-\bar{N})^{2}}{2 \omega_{\alpha} \bar{N}}\right]
$$

where the average number of particles $\bar{N}$ is defined by the energy and average volume, $\bar{N}=\left[\bar{V} E^{3} /\left(27 \pi^{2}\right)\right]^{1 / 4}$, and the scaled variance of the particle number distribution (98) equals,

$$
\omega_{\alpha}=\frac{1}{4}+\frac{1}{12} a_{V}^{2}
$$

The first term in the r.h.s. of Eq. (99) equals to the GMC scaled variance at fixed $E$ and $V$, the second term is due to the volume fluctuations. As it can be expected, $\omega_{\alpha}=\omega_{g m c}$ for $a_{V}=0$, and $\omega_{\alpha}=\omega_{p g m}$ for $a_{V}=1$. It also follows, $\omega_{g m c}<\omega_{\alpha}<\omega_{p g m}$ for $0<a_{V}<1$, and $\omega_{\alpha}>\omega_{p g m}$ for $a_{V}>1$. The $\alpha$-ensemble defined by Eqs. (97, 96) presents an extension of the GMC $\left(a_{V}=0\right)$ and PGM $\left(a_{V}=1\right)$ to a more general volume distribution.

In the second example both $E$ and $V$ are assumed to fluctuate. Equation (97) should be then extended as:

$$
P_{\alpha}(N)=\int d E d V P_{\alpha}(E, V) P_{g m c}(N ; E, V)
$$


First, uncorrelated volume and energy distributions are considered:

$$
P_{\alpha}(E, V)=P_{1}(V) \times P_{2}(E)
$$

where $P_{1}(V)$ is given by Eq. (96) and $P_{2}(E)$ is taken in the following form,

$$
P_{2}(E)=\left(2 \pi \omega_{E} a_{E}^{2} \bar{E}\right)^{-1 / 2} \exp \left[-\frac{(E-\bar{E})^{2}}{2 \omega_{E} a_{E}^{2} \bar{E}}\right]
$$

where $\bar{E}=3 T^{4} \bar{V} / \pi^{2}$ and $\omega_{E}=4 T$. A substitution of $P_{\alpha}(E, V)$ in Eq. (100) by the above distributions results in $P_{\alpha}(N)$ in the TL given again by the Gaussian (98) with the average number of particles $\bar{N}$ defined by the average energy and average volume, $\bar{N}=\left[\bar{V} \bar{E}^{3} /\left(27 \pi^{2}\right)\right]^{1 / 4}$, and the scaled variance equal to:

$$
\omega_{\alpha}=\frac{1}{4}+\frac{3}{4} a_{E}^{2}+\frac{1}{12} a_{V}^{2}
$$

The first term in the r.h.s. of Eq. (103) is the MCE scaled variance at fixed $E$ and $V$, the second term is due to energy fluctuations, and the third one comes from volume fluctuations. The results of the GMC, PGM, and GCE for the scaled variance of the particle number distribution can be obtained from Eq. (103) at $a_{V}=a_{E}=0 ; a_{V}=1, a_{E}=0 ;$ and $a_{V}=0, a_{E}=1$, respectively

Examples of correlated $V$ and $E$ distributions can be constructed as follows. One assumes the factorized form (101) with Eq. (102) for the energy distribution, but with $\bar{E}=3 T^{4} V / \pi^{2}$ and $\omega_{E}=4 T$, i.e. the average energy $\bar{E}$ depends on the volume $V$. Assuming,

$$
P_{1}(V)=\frac{1}{\bar{V}} \exp (-V / \bar{V})
$$

with $\bar{V}$ given by Eq. ([66), from Eqs. (101) and (102) with $a_{E}=1$, the results for the PGC are obtained.

The relation, $\bar{E}=3 T^{4} V / \pi^{2}$, can be used together with any form of the volume distribution $P_{1}(V)$. This yields a generalization of the GCE to the systems with externally given volume fluctuations. The energy fluctuations at fixed $V$ can be also selected in accordance with the physics requirements.

As the third example of the statistical ensembles with volume fluctuations one refers to the recent paper [17] where the micro-canonical ensemble with the scaling volume fluctuations for the ultra-relativistic ideal gas has been considered. The volume fluctuations were assumed to 
have the specific scaling properties. They were chosen to describe the KNO scaling [18] of the particle multiplicity distributions measured in proton-proton collisions at high energies. A striking feature of the model is power law form of the single momentum spectrum at high momenta, instead of the exponential Boltzmann distribution in the systems with fixed volume.

\section{SUMMARY}

In this paper the volume fluctuations in the statistical mechanics have been studied. The statistical ensembles with fixed external pressure were considered. Statistical systems of classical non-relativistic particles and non-interacting massless particles were discussed. The volume fluctuations in the pressure canonical ensemble become anomalously large in the thermodynamic limit for the first order phase transition, $\omega_{V} \propto N$, or at the critical point, $\omega \propto N^{1 / 2}$. Another type of anomalous volume fluctuations takes place for the pressure grand canonical ensemble. In this special ensemble, all thermodynamical variables are the intensive quantities, $\left(p_{0}, T, \mu\right)$. In the thermodynamical limit the mean particle multiplicity obtained within the considered ensembles with volume fluctuations is equal to the mean calculated within the micro-canonical, canonical, and grand canonical ensembles. This is not valid, however, for the scaled variance. The influence of the volume fluctuations in the pressure ensembles on particle number fluctuations have been discussed for ultra-relativistic ideal gas.

Following Ref. [16] the ensembles with volume fluctuations determined by the externally given distribution function were introduced. Multiplicity fluctuations are sensitive to the volume fluctuations. The volume fluctuations may also influence the behavior of the particle momentum spectra [17]. Thus, we believe that the concept of statistical ensembles with fluctuating extensive quantities, in particular, with fluctuating volume of the statistical system, may be appropriate for the statistical description of hadron production in relativistic collisions. It may be also useful for other physical systems. In fact, in all cases when the equilibrium statistical mechanics is used to calculate the fluctuations of the system properties.

\section{Acknowledgments}

We would like to thank D.V. Anchishkin, V.V. Begun, M. Gaździcki, W. Greiner, M. Hauer, B.I. Lev, I.N. Mishustin, O.N. Moroz, and Yu.M. Sinyukov for numerous discussions. This 
work was in part supported by the Program of Fundamental Researches of the Department of Physics and Astronomy of NAS, Ukraine.

[1] J. Cleymans, H. Oeschler, K. Redlich, and S. Wheaton, Phys. Rev. C 73, 034905 (2006); F. Becattini, J. Manninen, and M. Gaździcki, ibid. 73, 044905 (2006); A. Andronic, P. Braun-Munzinger, and J. Stachel, Nucl. Phys. A 772, 167 (2006).

[2] F. Becattini, Z. Phys. C 69, 485 (1996); F. Becattini and U. Heinz, Z. Phys. C 76, 269 (1997).

[3] J. Cleymans, K. Redlich, and E. Suhonen, Z. Phys. C 51, 137 (1991).

[4] M.I. Gorenstein, M. Gaździcki, and W. Greiner, Phys. Lett. B 483, 60 (2000).

[5] M.I. Gorenstein, A.P. Kostyuk, H. Stöcker, and W. Greiner, Phys. Lett. B 509, 277 (2001).

[6] F. Becattini and L. Ferroni, Eur. Phys. J. C 35, 243 (2004); 38, 225 (2004); V.V. Begun, L. Ferroni, M.I. Gorenstein, M. Gaździcki, F. Becattini, J. Phys. G 32, 1003 (2006); F. Becattini and L. Ferroni, Eur. Phys. J. C 51, 899 (2007); 52, 597 (2007).

[7] V.V. Begun, M. Gaździcki, M.I. Gorenstein, and O.S. Zozulya, Phys. Rev. C 70, 034901 (2004); V.V. Begun, M.I. Gorenstein, and O.S. Zozulya, Phys. Rev. C 72, 014902 (2005); A. Keränen, F. Becattini, V.V. Begun, M.I. Gorenstein, and O.S. Zozulya, J. Phys. G 31, S1095 (2005); F. Becattini, A. Keränen, L. Ferroni, and T. Gabbriellini, Phys. Rev. C 72, 064904 (2005); J. Cleymans, K. Redlich, and L. Turko, Phys. Rev. C 71, 047902 (2005); J. Phys. G 31, 1421 (2005); V.V. Begun and M.I. Gorenstein, Phys. Rev. C 73, 054904 (2006).

[8] V.V. Begun, M.I. Gorenstein, A.P. Kostyuk, and O.S. Zozulya, Phys. Rev. C 71, 054904 (2005).

[9] V.V. Begun, M.I. Gorenstein, A.P. Kostyuk, and O.S. Zozulya, J. Phys. G 32, 935 (2006).

[10] V.V. Begun, M.I. Gorenstein, M. Hauer, V.P. Konchakovski, and O.S. Zozulya, Phys. Rev. C 74, 044903 (2006);

[11] V.V. Begun, M. Gaździcki, M.I. Gorenstein, M. Hauer, B. Lungwitz, and V.P. Konchakovski, Phys. Rev. C 76, 024902 (2007).

[12] M. Hauer, V.V. Begun, and M.I. Gorenstein, arXiv:0706.3290 [nucl-th].

[13] M. Hauer, arXiv:0710.3938 [nucl-th].

[14] Yu.B. Rumer and M. Sh. Rivkin, Thermodynamics, Statistical Physics, and Kinetics (Nauka, Moscow, 1972) [in Russian]. 
[15] K.B. Tolpygo, Thermodynamics and Statistical Physics (Kiev University, 1966) [in Russian].

[16] M.I Gorenstein and M. Hauer, arXiv:08014219 [nucl-th].

[17] V.V. Begun, M. Gaździcki, and M.I. Gorenstein, arXiv:0804.0075 [hep-ph].

[18] Z. Koba, H. B. Nielsen, P. Olesen, Nucl. Phys. B 40, 317 (1972).

[19] L.D. Landau and E.M. Lifshitz. Statistical Physics (Course of Theoretical Physics, Volume 5), Pergamon Press, Oxford, 1980.

[20] M.I. Gorenstein, Yad. Fiz. 31, 1630 (1980) (Russ.); Sov. J. Nucl. Phys. 31, 845 (1980).

[21] Weisstein, Eric W. "Generalized Hypergeometric Function." From MathWorld-A Wolfram Web Resource. http://mathworld.wolfram.com/GeneralizedHypergeometricFunction.html

[22] A.P. Prudnikov, Yu.A. Brychkov, and O.I. Marichev, Integrals and Series, (Moscow, Nauka, 1986). 